\title{
DISCAPACIDAD EN CUIDADORES INFORMALES DE ADULTOS MAYORES CON ALTA DEPENDENCIA Y DIAGNÓSTICO DE ACCIDENTE CEREBROVASCULAR
}

\author{
DISABILITY IN INFORMAL CAREGIVERS OF ELDERLY PEOPLE WITH HIGH \\ DEPENDENCY AND DIAGNOSIS OF STROKE
}

\section{Camilo Patricio Poch Clavero' Eduardo Andrés Chacana Yorda ${ }^{2}$ Evelyn Angélica Álvarez Espinoza ${ }^{3}$ Catalina Ignacia Vásquez Avendaño ${ }^{4}$}

\begin{abstract}
RESUMEN
Los cuidadores de personas mayores con alta dependencia, en Chile se ven expuestos a demandas físicas y psicológicas complejas, situaciones de vulnerabilidad, escasa preparación y condiciones de precariedad, que desde la perspectiva de la Clasificación Internacional de la Funcionalidad puede ser una situación discapacitante por lo que se puede determinar el nivel de discapacidad de estos cuidadores informales.

Objetivo: Estimar la prevalencia y severidad de discapacidad, en cuidadores informales de personas mayores con situación de alta dependencia y con diagnóstico de accidente cerebro vascular.

Metodología: Estudio descriptivo y exploratorio, con muestra intencionada conformada por 32 cuidadores informales. Se evaluó la discapacidad en los cuidadores informales, mediante el cuestionario WHODAS II. Se estimó la prevalencia, severidad de discapacidad y descripción de variables contextuales y socio demográficas.

Resultados: La mediana de discapacidad global fue 23,9 [11,9-33,9]. Los dominios más comprometidos fueron relacionarse con otros, actividades domésticas y participación social. Todos los cuidadores informales presentaron dificultades, de ellos un 56,25\% presentó dificultades leves, un $37,5 \%$ dificultades moderadas y un 6,25\% severas. Los cuidadores informales pobres, los que declaran no distribuir tareas de cuidado, los con extensas jornadas laborales asociados al cuidado (mayor a 12 horas) y que cumplen el rol por más de 55 meses presentan mayores dificultades. Conclusión: Existe un alto porcentaje de discapacidad en cuidadores informales según el cuestionario WHODAS II. Los principales problemas se encuentran en los dominios actividades habituales, relacionarse con otros y participación social.
\end{abstract}

\section{PALABRAS CLAVES}

Cuidadores, persona mayor, accidente cerebro vascular

1 Kinesiólogo. Magister en Abordaje de Personas Mayores y Envejecimiento. Universidad Central de Chile. CESFAM Lo Valledor Norte. Av. Ecuador 4698 Departamento 301. CPI: 9170356 pochcamilo@gmail.com +56990743675

2 Kinesiólogo. Magister en Abordaje de Personas Mayores y Envejecimiento. Universidad Central de Chile. ELEAM Cordillera de los Andes Av. Lord Cochrane \# 417, Santiago Centro CP: 8330507 eduardochacanay@gmail.com+56990743675

3 Terapeuta Ocupacional Magister en Ciencias Biológicas con mención Neurociencias. Universidad De Chile. Facultad de Ciencias de la Salud, Universidad Central de Chile. Lord Cochrane \# 417, Santiago Centro 8330507 evelyn.alvarez@ucentral.cl +56 997413610

4 Licenciada en Terapia Ocupacional. Estudiante de Terapia Ocupacional. Facultad de Ciencias de la Salud, Universidad Central de Chile Lord Cochrane \# 417, Santiago Centro 8330507 Catalina.vasqueza@alumnos.ucentral.cl 


\section{ABSTRACT}

Caregivers of elderly people with high dependency in Chile are exposed to complex physical and psychological demands, situations of vulnerability, poor preparation and precarious conditions, situations that, from the perspective of the International Classification of Functionality, can be a disabling situation, so the level of disability of informal caregivers can be determined.

Aim: To estimate the prevalence and severity of disability, in informal caregivers of elderly people with a high dependency situation and a diagnosis of stroke.

Methodology: Descriptive and exploratory study, with an intentional sample made up of 32 ICs. Disability in informal caregivers was assessed by using the WHODAS II questionnaire. Prevalence, severity of disability, and description of contextual and socio-demographic variables were estimated.

Results: The median overall disability was 23.9 [11.9-33.9]. The most compromised domains were relating to others, domestic activities and social participation. All the informal caregivers presented difficulties, $56.25 \%$ of them presented mild difficulties, 37.5\% moderate difficulties and $6.25 \%$ severe. Poor informal caregivers, those who declare not to distribute care tasks, presenting long working hours associated with care (greater than 12 hours) and fulfill the role for more than 55 months, presented greater difficulties.

Conclusion: There is a high percentage of disability in informal caregivers according to the WHODAS II questionnaire. The main problems are found in the domains, habitual activities, relating to others and social participation.

\section{KEYWORDS}

Caregivers, elderly people, stroke.

Recibido:18/07/2020

Aceptado: 04/05/2021 


\section{INTRODUCCIÓN}

Actualmente la discapacidad se entiende desde un modelo biopsicosocial y basado en los criterios de la Clasificación Internacional de la Discapacidad y de la Salud (CIF) (Jiménez et al., 2012). Según la II Encuesta Nacional de Discapacidad en la población adulta, la prevalencia de discapacidad y severidad aumenta con la edad. Se destaca que la población adulta mayor un 20,8\% se encuentra en situación de discapacidad severa. Respecto a la prevalencia de dependencia en personas en situación de Discapacidad (PsD) es de un 40.4\% (SENADIS, 2016).

El accidente cerebro vascular (ACV) es la segunda causa de muerte en el mundo, con pérdida de años de vida saludable (Mathers \& Loncar, 2006). Quienes sobreviven al ACv pueden padecer alteraciones en estructuras y funciones corporales, limitación en las actividades y restricciones en la participación, secuelas que suelen ser permanentes y en los casos más severos pueden requerir apoyo parcial y total para realizar sus actividades básicas, instrumentales y avanzadas de la vida diaria (condición o situación de dependencia) (Mellon et al., 2015).

En muchos países incluido Chile la familia se responsabiliza en otorgar el principal soporte físico, mental y social de las personas secueladas de ACv. Los cuidadores informales $(\mathrm{Cl})$ de personas con diagnóstico de ACV deben enfrentar diversos tipos de problemas y necesidades según la etapa en que se encuentre la persona secuela de ACV (Godwin et al., 2013). Estos cuidados son fundamentados en relaciones afectivas, de parentesco familiar y con un fuerte componente de género, rasgos que caracterizan y afectan su visibilidad y reconocimiento social (APORTO, 2014; Centro Estudios de Vejez y Envejecimiento UC, 2015; Comunidad Mujer, 2014; López et al., 2016; Vaquiro \& Stiepovich, 2010).

En Chile el cuidado de personas en situación de discapacidad ( $\mathrm{PsD}$ ) con dependencia son realizados mayoritariamente por familiares mujeres. La familia es la primera institución que genera cuidados a PsD con dependencia y muchas veces sin ni siquiera tener algún entrenamiento o cursos acerca del cuidado, ni las competencias y habilidades para asumir con estas funciones (Centro Estudios de Vejez y Envejecimiento UC, 2015).
Los $\mathrm{Cl}$ de personas con ACV presentan cambios importantes en la vida cotidiana, causando además problemas de salud física y mental, la carga de cuidado de personas con ACv es alta en especial en el Cl (López et al., 2016; Troncoso, 2013).

Cuidar tiene repercusión en la esfera familiar directa (cuidador y cuidado) (Andersson et al., 2002; Troncoso, 2013), entornos cercanos y comunidad, pero también tiene consecuencias laborales, y en la sociedad en general (Flores et al., 2014; Larrañaga et al., 2008). Las principales consecuencias negativas del cuidado están relacionadas con la disminución de la calidad de vida (Crellin et al., 2014). Deterioro de su salud física y psicológica (Borg \& Hallberg, 2006; Pinquart \& Sörensen, 2007; Smith et al., 2014), limitaciones en la realización de actividades y restricción en la participación laboral (Lee \& Tang, 2015) y social (Zamarripa et al., 2017; Bergström et al., 2015).

Fox et al. (2002) introducen el paradigma de las discapacidades emergentes, entendidas éstas como producto de la ampliación de los conceptos tradicionales de discapacidad o asociadas a situaciones de salud recientemente identificadas. Esta nueva categoría de discapacidades derivadas de la relación exclusión social y pobreza con frecuencia enfrenta dificultades para su atención, debido a que pueden ser difíciles de reconocer o ser de corta duración y por tanto no encajan en la imagen tradicional de la PsD, y porque las políticas y servicios de atención se encaminan a las discapacidades tradicionales, con lo cual pueden no recibir la atención debida (Fox et al., 2002). Desde esta perspectiva "el cuidado" y sus consecuencias son situaciones potencialmente discapacitantes.

El objetivo del estudio es evaluar la prevalencia y severidad de dificultades y problemas en la realización de actividades cotidianas y de participación social (discapacidad) través de la evaluación de discapacidad con el cuestionario para la Evaluación de la Discapacidad de la Organización Mundial de la Salud (WHODAS II), en Cl de personas mayores con situación de alta dependencia y con diagnóstico de Accidente Cerebro Vascular. 


\section{MATERIALES y MÉTOdoS}

El tipo de estudio fue de carácter cuantitativo con alcance descriptivo no experimental. La muestra fue por conveniencia, se aplicó el cuestionario WHODAS II a 32 los $\mathrm{Cl}$ de PsD con alta dependencia con diagnóstico de ACV que pertenezcan a los centros de salud de la comuna de Pedro Aguirre Cerda.

\section{Criterios de inclusión}

Ser $\mathrm{Cl}$ de una persona mayor con alta dependencia, secuelada de ACV perteneciente a algunos de los programas de alta dependencia de los centros de salud de la comuna de Pedro Aguirre Cerda, ser mayor de 18 años, cumplir el rol de cuidador a lo menos con un mes de antigüedad y cuidar a personas mayores con diagnóstico de ACv con un nivel de dependencia severa o total según el Índice de Barthel (Barthel menor a 35 pts.).

\section{Criterios de exclusión}

Ser $\mathrm{Cl}$ con antecedentes de discapacidad previa (Registro Nacional de discapacidad vigente o en trámite) y percibir remuneración por conceptos de cuidado de la persona mayor (excluye recibir estipendio).

\section{Proceso de reclutamiento}

Se realizaron las gestiones técnicas correspondiente con el departamento de salud de la comuna de Pedro Aguirre Cerda y los encargados de los Programas de alta dependencia de cada centro de salud para poder acceder a los registros de las personas en situación de dependencia, con $\mathrm{Cl}$, que cumplieran con los criterios de inclusión y exclusión. Posteriormente se invitó a los $\mathrm{Cl}$ a participar de la investigación, mediante llamados telefónicos. A los $\mathrm{Cl}$ que desearon participar se acordó según disponibilidad una visita domiciliaria para realizar cuestionarios WHODAS II y registro de antecedentes generales. La recolección de datos se obtuvo desde junio a agosto del 2019.

\section{Variables del estudio}

La variable dependiente es la Discapacidad de los cuidadores, la cual será medida mediante el cuestionario WHODAS II. La variable independiente es la condición de dependencia de las personas mayores con diagnóstico de ACV, la cual fue medida mediante el índice de Barthel, cuyos resultados deben ser iguales o inferiores a 35 pts. Además, se considerarán variables contextuales que pudiesen incidir en el nivel de discapacidad de los Cl.

\section{Instrumentos de recolección de datos}

Los antecedentes generales (variables contextuales) se extrajeron al momento de la aplicación de cuestionario WHODAS II y la situación de discapacidad de los $\mathrm{Cl}$ fue otorgada por la evaluación con el cuestionario WHODAS II Versión de 36 preguntas aplicadas por un entrevistador. WHODAS II brinda una métrica común del impacto de cualquier condición de salud en términos del funcionamiento con una escala de o-100, donde o puntos es nada de discapacidad y 100 totalmente discapacitado.

WHODAS II propone al igual que la CIF como primer calificador genérico con escala negativa, para indicar la extensión o magnitud de la deficiencia, una dificultad o una barrera: Ninguna (0-4\%), Leve (5-24\%), Moderada $(25-49 \%)$, Severo $(50-95 \%)$ y extremo/no puede realizarlo (96-100\%).

\section{Consideraciones Éticas}

Esta investigación consideró los criterios propuestos por Emanuel E. para que una investigación clínica sea ética (Emanuel, 1999). Este estudio fue aprobado por el comité de ética de la Universidad Central de Chile y el Comité de Ética Científico del Servicio de Salud Metropolitano Sury el patrocinio del Departamento de Salud de la comuna de Pedro Aguirre Cerda. 
Los participantes de este estudio firmaron un consentimiento informado para participar de esta investigación y autorizaron la publicación de los resultados obtenidos de forma general y no individualizada.

\section{Técnica de Análisis de estadístico}

A todas las variables del estudio se les aplicará un análisis estadístico descriptivo, utilizando proporciones promedio (desviación estándar), mediana (p25-p75), según corresponda previo análisis de normalidad y homocedasticidad mediante las pruebas Wilk y Levene.

\section{Resultados}

Se evaluó a $32 \mathrm{Cl}$ con un promedio de edad de 58,7 (DS: 12.5 años). En la tabla 1 se muestran los antecedentes sociodemográficos de los $\mathrm{Cl}$. Respecto a los adultos mayores en situación de alta dependencia 21 son mujeres y 11 son hombres, su edad promedio es de 78.1 años (DS: 9.5 años). Un 37\% tiene nivel de dependencia severa y un $62.5 \%$ total. En relación a su parentesco con su cuidador/a informal es: $53 \%$ son hijos/as, 25\% Pareja, $13 \%$ hermano/a, $3 \%$ otro familiar y $6 \%$ otra persona.

\section{TABLA 1 CARACTERIZACIÓN SOCIO DEMOGRÁFICA DE LOS CUIDADORES INFORMALES}

\begin{tabular}{|c|c|c|}
\hline Variable & $\mathrm{N}$ & $(\%)$ \\
\hline \multicolumn{3}{|l|}{ Sexo } \\
\hline Mujeres & 28 & 87,5 \\
\hline Hombres & 4 & 12,5 \\
\hline \multicolumn{3}{|l|}{ Estado Civil } \\
\hline Casado/a o viviendo en pareja & 12 & 46,87 \\
\hline solteros/as & 10 & 31,25 \\
\hline Divorciado/a o separado/a & 5 & 15,63 \\
\hline Viudo/a & 2 & 6,25 \\
\hline \multicolumn{3}{|l|}{ Situación ocupacional } \\
\hline Trabajo remunerado & 4 & 12,5 \\
\hline Estudiante & 2 & 6,25 \\
\hline Desempleado & 10 & 31,25 \\
\hline Dueño/a de casa & 10 & 31,25 \\
\hline Jubilado/a & 6 & 18,75 \\
\hline \multicolumn{3}{|l|}{ Situación de Pobreza } \\
\hline Sobre la línea de la pobreza & 6 & 18.75 \\
\hline Bajo la línea de la pobreza & 18 & 56,25 \\
\hline Pobreza extrema & 8 & 25 \\
\hline \multicolumn{3}{|l|}{ Años de Educación Formal } \\
\hline$<8$ años & 12 & 37,5 \\
\hline 8-12 años & 16 & 50 \\
\hline$>12$ años & 4 & 12,5 \\
\hline
\end{tabular}


En relación a la frecuencia de problemas reportados por el cuidador informal secundarios al Acv el $97 \%$ de los adultos mayores con dependencia tiene problemas severos para movilizarse o trasladarse, un $75 \%$ problemas de deglución, un $69 \%$ problemas cognitivos, un $59 \%$ problemas de comunicación, un $56 \%$ problemas del comportamiento y un $25 \%$ presentó úlceras por presión que requieren de cuidados de enfermería. Un 16\% de las personas dependientes usa Sonda Foley, un $9 \%$ Sonda Nasogástrica o Gastrostomía y un 6\% Traqueotomía.

El tiempo promedio a cargo del cuidado de la persona en situación de alta dependencia es de 4.5 años (DS: 3,8 años, $\mathrm{P}_{50} \mathrm{O} 3$ años), dedicando en promedio 14.1 horas al día al cuidar (cuidados directos e indirectos)

La razón más frecuente por los $\mathrm{Cl}$ que asumen el rol de cuidador es "era la única persona que podía cuidarlo" con un $81.25 \%$. Solo $25 \%$ de los Cl declaró que co-distribuyen el rol del cuidador (directos o indirectos) con alguna otra persona de su red de apoyo (turnos rotativos diarios o durante jornadas de mañana o tarde).

La distribución de los resultados del cuestionario WHODAS II fue no paramétricas, para esta muestra la mediana de la discapacidad global fue de 23,9 (11,933,9) los Dominios con mayores problemas fueron: "Participación social", "Actividades habituales" y "Relacionarse con otros". El resumen de los principales resultados se muestra en la tabla 2.

Un $56 \%$ de los $\mathrm{Cl}$ presentan dificultades leves, un $38 \%$ dificultades moderadas y un $6 \%$ problemas severos en relación a su puntaje de Discapacidad Global del Cuestionario WHODAS II.

El resumen de la frecuencia relativa de dificultades por severidad y dominio se muestra en la tabla 3. Se destaca el alto porcentaje de $\mathrm{Cl}$ que presentan problemas moderados a extremos en los dominios "Participación social" (69\%), "Actividades habituales o domésticas" $(45 \%)$ y relacionarse con otros $(44 \%)$.

\section{Discusión}

Este estudio exploró las consecuencias negativas asociadas al rol del cuidado en particular de su nivel de discapacidad. Para esta muestra de $\mathrm{Cl}$ de personas adultas mayores en situación de alta dependencia y diagnóstico de ACv en la comuna de Pedro Aguirre Cerda la mediana de la discapacidad global fue de 23,9, (discapacidad leve según WHODAS II). Los dominios más comprometidos fueron "Relacionarse con otros", "Actividades Domésticas", "Participación Social" y "Actividades laborales o educacionales" donde solo $6 \mathrm{Cl}$ respondieron este ítem.

Llama la atención el alto porcentaje de $\mathrm{Cl}$ que presentan dificultades moderadas (38\%) y severas (6\%), datos mayores a los reportados por la ENDISC II en población adulta a nivel nacional (SENADIS, 2016). Si bien ambos estudios ocupan metodologías diferentes, ambos utilizan criterios establecidos por la CIF para valorar la discapacidad.

En relación a la caracterización de los $\mathrm{Cl}$ encontramos que en nuestro estudio el promedio de edad de los $\mathrm{Cl}$ fue de 58.7 años y el rango etario más frecuente es de los 40 a 59 años datos que son similares a los reportados por SENADIS (2016).

La feminización del cuidado en esta muestra fue un $87.5 \%$. Estos hallazgos se condicen con los estudios realizados en población rural, urbana y en distintas zonas geográficas del país confirman que los $\mathrm{Cl}$ son principalmente familiares mujeres (APORTO, 2014; Jofré \& Sanhue$\mathrm{za}, 2 \mathrm{O10}$; Troncoso, 2013)

En relación al nivel socioeconómico un 81.25\% de los Cl evaluados declaró ingresos per-cápita bajo la línea de la pobreza. Esta situación es crítica ya que si le sumamos los altos costos directos e indirectos del cuidado probablemente los $\mathrm{Cl}$ y/o sus familias no alcanzan a cubrir los gastos que genera la condición de salud de la persona con dependencia, datos que si bien no fueron valorados en esta investigación podría ser mucho mayor a los reportado por Durán y Rogero (Duran \& Rogero, 2004)

El tiempo dedicado a cuidar en promedio es de 4,5 años y las jornadas de cuidado diarios se extienden en promedio a 14,1 horas diarias. La razón más frecuente para asumir el rol de cuidador el ser la única persona que podía cuidarlo con un $81.25 \%$ datos similares a 


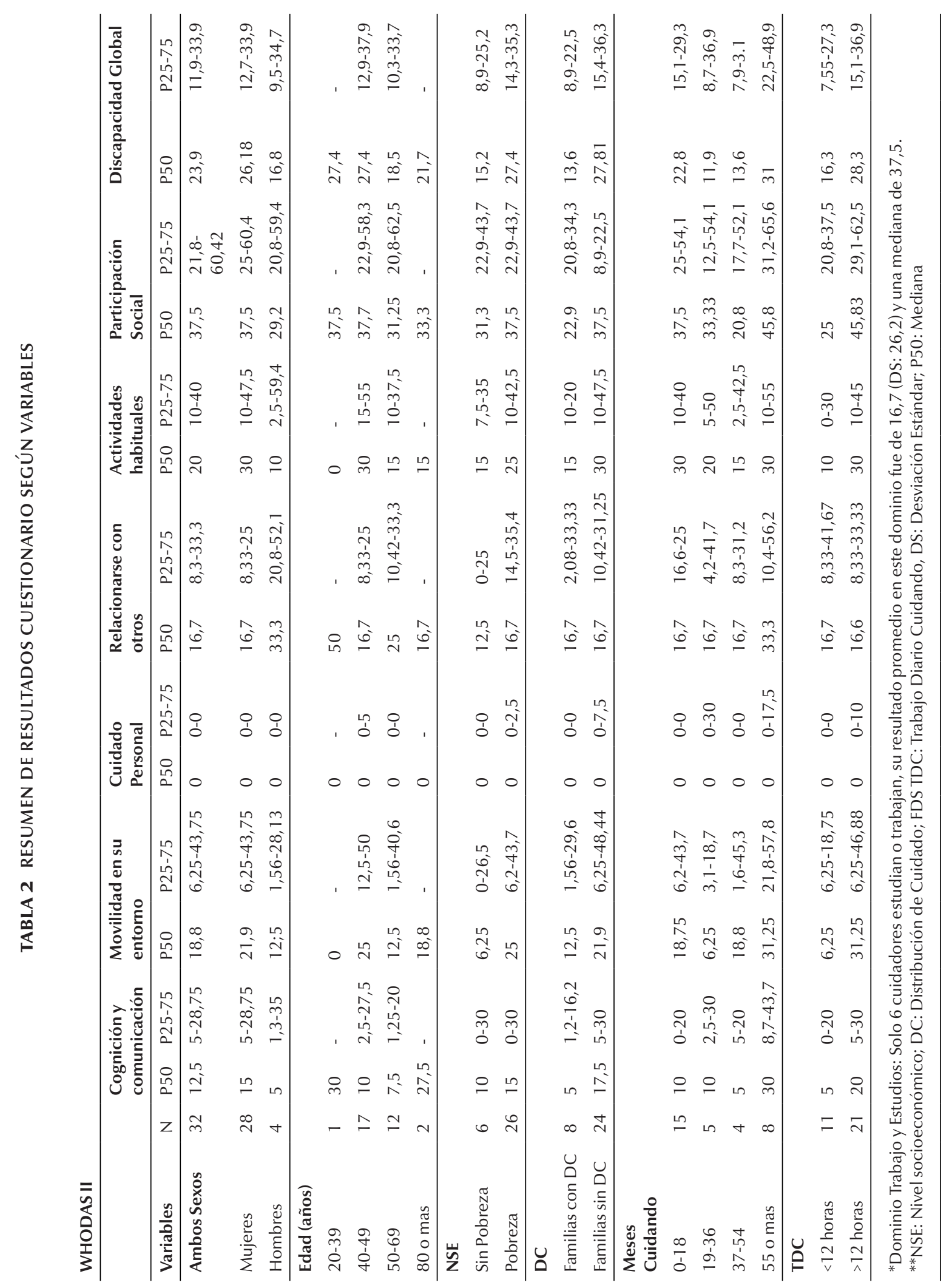


TABLA 3 PORCENTAJE DE DIFICULTADES Y SEVERIDAD POR DOMINIO DEL CUESTIONARIO WHODAS II

\begin{tabular}{lllllll} 
Dominio WHODAS II & \multicolumn{7}{l}{ Categoría* } \\
\hline & Ninguna & Leve & Moderado & Severo & Extremo & No Aplica \\
\hline Cognición y Comunicación & $22 \%$ & $44 \%$ & $31 \%$ & - & $3 \%$ & - \\
Movilidad en su entorno & $16 \%$ & $38 \%$ & $28 \%$ & $19 \%$ & - & - \\
Cuidado Personal & $81 \%$ & $16 \%$ & - & $3 \%$ & - & - \\
Relacionarse con otros & $9 \%$ & $47 \%$ & $28 \%$ & - & - & - \\
Actividades habituales & $16 \%$ & $38 \%$ & $25 \%$ & $22 \%$ & - & - \\
Trabajo o estudios & $13 \%$ & - & $3 \%$ & $3 \%$ & - & $81 \%$ \\
Participación Social & - & $25 \%$ & $38 \%$ & $31 \%$ & - & - \\
\hline Discapacidad Global & - & $56 \%$ & $38 \%$ & $6 \%$ & - & - \\
\hline
\end{tabular}

*Ninguna (0-4\%), Leve (5-24\%), Moderada (25-49\%), Severo (50-95\%) y extremo/no puede realizarlo (96-100\%).

No aplica: Cuidadores no realiza actividades relacionados trabajo remunerado o estudios

los reportados por la literatura a nivel nacional (SENAMA, 20O9; APORTO, 2014; Centro de Estudios de Vejez y Envejecimiento UC, 2015)

De este modo los $\mathrm{Cl}$ y sus familias experimentan una gran vulnerabilidad económica, en la medida que deben destinar gran parte de los recursos en pos de la persona dependiente, a la vez que se ven enfrentados a problemas de productividad y de compatibilidad con los roles laborales y del hogar (Troncoso, 2013). Además, un 37,5\% de los $\mathrm{Cl}$ tiene 8 años o menos de educación formal lo que aumenta su vulnerabilidad social y disminuye sus oportunidades de empleos formales. Resultados similares a los reportados por García et al donde los $\mathrm{Cl}$ son mujeres, pobres, con bajo nivel educacional y sin empleos formales (García et al., 2004).

En relación a la restricción para realizar actividades laborales (remuneradas), educacionales y actividades sociales puede ser atribuible principalmente las extensas jornadas dedicadas al cuidado directo e indirecto y la no distribución de cuidados de la PSD dependientes, este contexto es relevante ya que puede aumentar la vulnerabilidad y exclusión social de los $\mathrm{Cl}$, especialmente en aquellos que están en edad laboral (APORTO, 2014; Lee \& Tang, 2015)
Llama la atención que del total de los cuidadores entrevistados, solo 4 de ellos $(12,5 \%)$ se encontraran realizando actividades remuneradas, estas principalmente como trabajadores independientes o en actividades informales, asociadas a tiempos libres durante sus extensas jornadas de cuidados, generando condiciones de inserción laboral desfavorables (Lee \& Tang, 2015)

Respecto a las dificultades en el dominio "relacionarse con otros" en nuestra muestra alcanza un 90,63\%. Estas son relevantes ya que pueden ser debido al aislamiento social y escasa ayuda que reciben los $\mathrm{Cl}$.

En términos generales los $\mathrm{Cl}$ de personas dependientes, tanto de personas mayores como población en general, poseen poco tiempo libre para poder descansar, hacer otras actividades de su interés o bien compartir con personas cercanas incluso con sus parejas al igual que describe Troncoso (Troncoso, 2013), situación que es relevante si consideramos que la soledad y el aislamiento son los elementos que influyen más negativamente en el concepto subjetivo de bienestar (Grigorovich et al., 2015) y limitan la posibilidad de establecer y mantener relaciones con otras personas (Troncoso, 2013).

En relación al dominio "actividades habituales" los $\mathrm{Cl}$ reportaron un $45 \%$ de dificultades en este dominio. En términos generales la literatura coincide en que los 
$\mathrm{Cl}$ tienen poco tiempo para realizar actividades de su interés incluso en los casos en que los $\mathrm{Cl}$ señalan tener tiempo para hacer otras actividades estas se vinculan indirectamente al cuidado (Centro Estudios de Vejez y Envejecimiento UC, 2015).

Estas restricciones y limitaciones en la realización de actividades habituales pueden tener una relación directa con la satisfacción que ellos tienen de su vida y la realización de actividades relacionadas con el trabajo doméstico (Zamarripa et al., 2017; Bergström et al., 2015).

En cuanto a dificultades para realizar actividades de participación social un $62,5 \%$ de los $\mathrm{Cl}$ presentan dificultades severas a extremas, en especial los $\mathrm{Cl}$ que no distribuyen las tareas de cuidado con algún miembro de la familia, los que ejercen el rol por más de 55 meses y las con alta carga de trabajo (más de 12 horas diarias) datos que son similares a los reportados Grigorovich A. et al (Grigorovich et al., 2015) en el cual reporta que los cuidadores jóvenes, con síntomas depresivos, las trabajadoras formales, los con alta carga laboral y cuidar a una persona con déficit cognitivos tienen mayor riesgo de restricción en la participación.

Los resultados de discapacidad global entregados por el cuestionario WHODAS II en $\mathrm{Cl}$ de adultos mayores en situación de dependencia severa y con diagnóstico de ACV son similares a los reportados en población brasileña con dolor de origen músculo esquelético (Silva et al., 2013) y en población argentina con problemas de salud mental (Gómez et al., 2015)

La situación de pobreza de los $\mathrm{Cl}$, las largas jornadas de cuidado (12 horas o más), la carga física y mental del rol del cuidador y la escasa colaboración para distribuir las tareas de cuidado son variables que pueden repercutir en el nivel de en la discapacidad y en particular en la participación social de los $\mathrm{Cl}$ (Grigorovich et al., 2015)

\section{Limitaciones}

Una de las limitaciones de esta investigación fue una muestra de solo $32 \mathrm{Cl}$. Esto pudo influir en los resultados esperados sobre las consecuencias negativas asociadas al cuidado en particular sobre su nivel de discapacidad global y su correlación con otras variables sociodemográficas y contextuales.
Si bien los $\mathrm{Cl}$ presentaron gran cantidad de dificultades en particular en los dominios "relacionarse con otros", "actividades habituales" y "participación social" la metodología no permite generalizar los resultados encontrados. Además, este estudio no consideró las condiciones de salud de los $\mathrm{Cl}$ que podrían incidir en el nivel de discapacidad al igual que la heterogeneidad de variables familiares, ambientales y del rol del cuidador.

Es necesario adaptary validar el cuestionario WHODAS II en población chilena y cuidadores para proporcionar datos normativos.

\section{Proyección del estudio}

Se sugiere realizar líneas de investigación sobre las potenciales consecuencias negativas asociadas al cuidado de adultos mayores en situación de alta dependencia y diagnóstico de ACV y con otros diagnósticos como demencia, enfermedad de Parkinson u otras enfermedades altamente prevalentes en la población chilena.

Estudios de seguimiento de casos podrían ser útiles para visualizar el curso natural de las posibles consecuencias negativas asociadas al cuidado en particular de la funcionalidad del $\mathrm{Cl}$, estimar el grado de reversibilidad de estas dificultades y valorar el efecto de intervenciones socio sanitario en esta población.

La realización de perfiles de funcionamiento de $\mathrm{Cl}$ en base a los criterios de la CIF permitiría mejorar la gestión e intervenciones socio sanitarias en esta población con alta vulnerabilidad social y que hoy está siendo sub-atendida en relación a sus múltiples necesidades y dificultades que presentan.

Es relevante realizar otras investigaciones complementarias en los ámbitos de salud, la participación social, de género y familia a fin de profundizar y elaborar estrategias que permitan el abordaje integral del $\mathrm{Cl}$.

\section{Implicancias Profesionales}

Los resultados principales de esta investigación contribuyen a validar el rol de los $\mathrm{Cl}$ y las consecuencias negativas asociadas a este, en particular de la discapacidad. Además de determinar su impacto de manera cuantificable 
Este conocimiento aporta a comprender el rol del $\mathrm{Cl}$ desde la perspectiva de funcionamiento humano (CIF) y fortalecer la gestión clínica en esta población.

De este modo la información proporcionada por esta investigación puede contribuir para orientar las políticas públicas e intervenciones socio sanitarias en $\mathrm{Cl}$ de personas mayores en situación de alta dependencia causado por un ACV, considerando el envejecimiento poblacional y el aumento de enfermedades crónicas en la población adulta mayor.

Proponemos que los servicios socio sanitarios para los cuidadores y personas en situación de alta dependencia deben orientarse a:

- Proteger el acceso a la salud del cuidador, la persona en situación de alta dependencia y su familia.

- Apoyar la participación social de los cuidadores, proteger el bienestar de los cuidadores a largo plazo y asegurar la capacidad del cuidador para brindar atención a los secuelados de ACV.

- Favorecer la distribución de roles de cuidado en la familia para mitigar la alta carga de trabajo del cuidador.

- Resguardar los derechos de los $\mathrm{Cl}$ a la recreación y ocio, vida sexual y afectiva, participación social, vida laboral, entre otros.

Fortalecer el apoyo de asistente personal (cuidadores formales), la distribución de roles de cuidados en las familias, y el apoyo de los centros de salud y comunidad al cuidador principal (y sus necesidades) pueden ser estrategias útiles para disminuir la discapacidad de los cuidadores.

\section{Conclusiones}

Ser $\mathrm{Cl}$ de una persona mayor con alta dependencia y diagnóstico de Accidente cerebro vascular en la comuna de Pedro Aguirre Cerda puede ser una situación discapacitante. Los $\mathrm{Cl}$ de la muestra presentaron altos niveles de discapacidad en particular en los dominios "Relacionarse con otros", "Actividades habituales o domésticas", "Actividades laborales" y "Participación social" del cuestionario WHODAS II.

La mediana de la discapacidad global encontrada en esta investigación fue de 23,9 [11.9-33.9]. Según nivel de discapacidad un 56.25\% (18) de los Cl presenta dificultades leves, un $37,5 \%$ (12) dificultades moderadas y un $6,25 \%$ (2) dificultades severas.

Los $\mathrm{Cl}$ en situación de pobreza, las extensas jornadas de cuidado, la carga física y mental en el desempeño del rol del cuidador y la escasa colaboración para distribuir las tareas de cuidado son condiciones que pueden incidir negativamente en el nivel de discapacidad y restricción en su participación social.

Las personas que cumplen el rol de cuidador son mayoritariamente familiares mujeres, pobres, con bajo nivel educacional y con empleos precarios aumentando su vulnerabilidad y exclusión social.

Pueden ser estrategias útiles para disminuir la discapacidad de los Cl: el fortalecimiento de los programas de cuidados de larga duración, la distribución de roles de cuidados en las familias y comunidad, y la protección y resguardo de la salud del cuidador por parte de los centros de salud.

Para futuras investigaciones es necesario adaptar y validar el cuestionario WHODAS II en población chilena para proporcionar datos normativos.

\section{Agradecimientos}

Al Departamento de Salud de la Comuna de Pedro Aguirre Cerda por su patrocinio para realizar esta investigación.

\section{RefERENCIAS BibLIOGRÁfiCAS}

Andersson, A., Levin, L. A. \& Emtinger, B. G. (2002). The Economic Burden of Informal Care. International journal of technology assessment in health care, 18(1): 46-54.

APORTO, (2014). Diseño de una estrategia para la reducción de brechas entre la demanda de familias en situación de vulnerabilidad por contar con integrantes en condiciones de dependencia (derivadas del envejecimiento, enfermedad y/o discapacidad) y la oferta intersectorial de servicio de cuidados.

Bergström, A. L., von Koch, L., Andersson, M., Tham, K. \& Eriksson, G. (2015). Participation in Everyday Life and Life Satisfaction in Persons with Stroke and Their Caregivers 3-6 months after Onset. Journal of Rehabilitation Medicine, 47(6): 508-515. 
Borg, C. \& Hallberg, I. R. (2006). Life Satisfaction among Informal Caregivers in Comparison with Non-Caregivers. Scandinavian Journal of Caring Sciences 20(4): 427-438.

Centro Estudios de Vejez y Envejecimiento UC. (2015). "Estudio Sistematización y Descripción de los Perfiles de las Cuidadoras de Personas Dependientes, las Demandas de Apoyo que las Cuidadoras Presenten y los Programas Existentes para Aliviar el Trabajo de Cuidado". Instituto de Sociología, Facultad de Ciencias Sociales: 1-76.

Comunidad mujer. (2014). Modelos de Cuidado de Personas Adultas Dependientes, Cuidadoras y Su Participación en el Mercado Laboral. http://www.comunidadmujer.cl/biblioteca-publicaciones/ wp-content/uploads/2014/07/Informe-Final-Modelos-decuidado-cuidadoras-y-participación.pdf

Crellin, N. E., Orrell, M., McDermott, O. \& Charlesworth, G. (2014). Self-Efficacy and Health-Related Quality of Life in Family Carers of People with Dementia: A Systematic Review. Aging and Mental Health 18(8): 954-969. http://dx.doi.org/10.1080/136 07863.2014.915921.

Durán M. A. \& Rogero J. (2004). Nuevas Parejas para Viejas Desigualdades. Revista de Estudios de Juventud (67): 25-37.

Emanuel, E. (1999). ¿Qué hace que la Investigación Clínica sea Ética? Siete Requisitos Éticos. Pautas Éticas de Investigación en Sujetos Humanos: Nuevas Perspectivas: 82-93.

Flores, N., Jenaro, C., Moro, L. \& Tomşa, R. (2014). Health and Quality of Life of Family and Professional Caregivers of Dependent Elderly: A Comparative Study. European Journal of Investigation in Health, Psychology and Education 4(2): 79-88.

Fox, M. H., Kim, K. \& Ehrenkrantz, D. (2002). Statewide Disability Information Systems. Journal of Disability Policy Studies 13(3): 171-179.

García-Calvente, M., Mateo-Rodríguez, I. \& Eguiguren, A. (2004). The System of Informal Caregiving as Inequality. Gaceta sanitaria / S.E.S.P.A.S 18 Suppl 1(January 2014): 132-139.

Godwin, K. M., Swank, P. R., Vaeth, P. \& Ostwald, S. K. (2013). The Longitudinal and Dyadic Effects of Mutuality on Perceived Stress for Stroke Survivors and Their Spousal Caregivers. Aging and Mental Health 17(4): 423-431.

Gomez, P., Enders, J. E., Alvarado, R., Cometto, M. C., \& Fernández, A. R. (2015). Psychological evaluations of operation of patients with mental disorders. Revista de la Facultad de Ciencias Médicas (Cordoba, Argentina), 72(4), 243-249.

Grigorovich, A., Forde, S., Levinson, D., Bastawrous, M., Cheung, A. M. \& Cameron, J. I. (2015). Restricted Participation in Stroke Caregivers: Who is at Risk?. Archives of Physical Medicine and Rehabilitation, 96(7): 1284-1290

Jimenez, M. T., Gonzalez, P. \& Martin, J. M. (2012). La clasificación internacional del funcionamiento, de la discapacidad y de la salud (CIF) 2001. Revista Española de Salud Pública 32(3): 59-60.

Jofré, V. \& Sanhueza, O. (2010). Evaluación de La Sobrecarga de Cuidadoras/es Informales. Ciencia y Enfermería 16(3): 111-120.
Larrañaga, I., Martin, U., Bacigalupe, J. M., Begisristáin, M., Valderrama, M. J. \& Arregi, B. (2008). Impacto Del Cuidado Informal En La Salud y La Calidad de Vida de Las Personas Cuidadoras: Análisis de Las Desigualdades de Género. Gaceta Sanitaria 22(5): 443-450.

Lee, Y. \& Tang, F. (2015). More Caregiving, Less Working: Caregiving Roles and Gender Difference. Journal of Applied Gerontology 34(4): 465-483.

López, L., Castellanos, F. \& Muñoz, E. (2016). Cuidado Popular de Famílias Com Idoso Sobrevivente Do Primeiro Acidente Vascular Cerebral. Aquichan 16(2): 169-178.

Mathers, C. D., \& Loncar, D. (2006). Projections of Global Mortality and Burden of Disease from 2002 to 2030. PLoSMedicine 3(11): 2011-30

Mellon, L., Brewer, L., Hall, P., Horgan, F., Williams, D., Hickey, A. \& ASPIRE-S group. (2015). Cognitive Impairment Six Months after Ischaemic Stroke: A Profile from the ASPIRE-S Study. BMC Neurology 15(1): 1-9.

Pinquart, M. \& Sörensen, S. (2007). Correlates of Physical Health of Informal Caregivers: A Meta-Analysis. Journals of GerontologySeries B Psychological Sciences and Social Sciences 62(2): 126-137.

SENADIS. (2016). II Estudio Nacional de Discapacidad 2015. Santiago.

SENAMA. (2009). Estudio Nacional de Dependencia en Personas Mayores. Santiago.

Silva, C., Coleta, I., Silva, A., Amaro, A., Alvarelhão, J., Queiros, A. \& Rocha, N. (2013). Adaptation and Validation of Whodas 2.0 in Patients with Musculoskeletal Pain. Revista de Saúde Pública 47(4): 1-6.

Smith, L., Onwumere, J., Craig, T., McManus, S., Bebbington, P. \& Kuipers E. (2014). Mental and Physical Illness in Caregivers: Results from an English National Survey Sample. British Journal of Psychiatry 205(3): 197-203.

Troncoso, D. (2013). Cuidadores Informales de Personas Dependientes En Chile. Aspectos Psicosociales y Culturales. (Tesis doctoral) .Universidad de Salamanca. http://gredos.usal.es/jspui/bitstream/10366/124233/1/DSC_TroncosoMirandaDeizaYanet_Tesis. pdf.

Vaquiro, S. \& Stiepovich. J. (2010). Cuidado Informal, Un Reto Asumido Por La Mujer. Ciencia y enfermería 16(2): 9-16.

Zamarripa, E. A., Tamez, B. M. \& Ferreira, M. R. (2017). Repercusiones Del Cuidado Informal En La Vida Laboral y Personal de Las Mujeres Cuidadoras. Azarbe 9(6): 47-56.

\section{Financiamiento}

Este estudio no contó con financiamiento de terceros. 